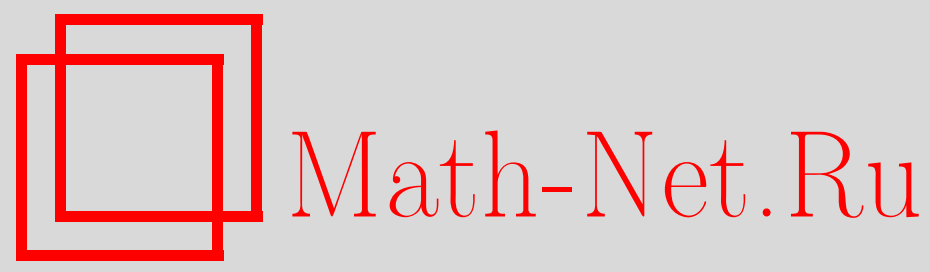

М. А. Приходько, Асимптотика информационной энтропии для двумерного аналога релятивистского атома водорода в модели Козлова-Никишина, Матем. заметки, 2005, том 78, выпуск 5, 727-744

DOI: https://doi.org/10.4213/mzm2636

Использование Общероссийского математического портала Math-Net.Ru подразумевает, что вы прочитали и согласны с пользовательским соглашением http://www.mathnet.ru/rus/agreement

Параметры загрузки:

IP : 18.234 .197 .8

26 апреля 2023 г., $17: 32: 11$

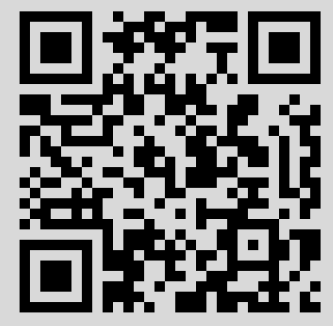




\title{
АСИМПТОТИКА ИНФОРМАЦИОННОЙ ЭНТРОПИИ ДЛЯ ДВУМЕРНОГО АНАЛОГА РЕЛЯТИВИСТСКОГО АТОМА ВОДОРОДА В МОДЕЛИ КОЗЛОВА-НИКИШИНА
}

\author{
М.А. Приходько
}

\begin{abstract}
Для двумерной лоренц-инвариантной модели атома водорода получены волновые функции связанных состояний в координатном представлении, а для невозбужденных по времени состояний и в импульсном представлении. Для них исследована коротковолновая асимптотика информационной энтропии.

Библиография: 12 названий.
\end{abstract}

1. Введение. Состояние квантово-механической системы описывается волновой функцией $\Psi(\vec{q})$, определенной в конфигурационном пространстве и удовлетворяющей уравнению Шрёдингера

$$
\widehat{H} \Psi=E \Psi,
$$

где самосопряженный оператор $\widehat{H}$ - это гамильтониан системы, а $E$ - ее энергия. Уравнение Шрёдингера служит выражением закона сохранения энергии.

Связанным состояниям системы соответствуют интегрируемые с квадратом решения этого уравнения. Тогда $|\Psi|^{2}$ представляет собой плотность распределения частищ в конфигурационном пространстве (после соответствующей нормировки).

Переход к импульсному представлению $\Psi(\vec{q}) \rightarrow \Psi^{*}\left(\overrightarrow{q^{*}}\right)$ осуществляет преобразование Фурье. Важную роль во всей квантовой механике играет принцип неопределенности Гейзенберга, который в безразмерных величинах имеет вид

$$
\Delta q \cdot \Delta q^{*} \geqslant \frac{1}{2}
$$

где $q$ - одна из обобщенных координат,

$$
(\Delta q)^{2}=\left\langle(q-\langle q\rangle)^{2}\right\rangle
$$

и аналогичное выражение справедливо для $\Delta q^{*}$. При этом

$$
\langle q\rangle=\int q|\Psi|^{2} d \vec{q}
$$


С другой стороны для изучения меры неопределенности плотности вероятности используется так назьваемая информационная энтропия Больцмана-Шеннона

$$
S_{\Psi}=-\int \ln |\Psi|^{2} \cdot|\Psi|^{2} d \vec{q} .
$$

В работе [1] было доказано неравенство

$$
S_{\Psi}+S_{\Psi^{*}} \geqslant(\ln \pi+1) \cdot \mathfrak{D}
$$

где $\mathfrak{D}$ - размерность конфигурационного пространства.

Это неравенство также описывает неопределенность квантово-механической системы и возможно является более точной мерой неопределенности.

Исследование энтропии и энтропийного соотношения неопределенности различных систем привлекает все большее внимание.

Существует множество работ, написанных на эту тему (см., например, [2]-[5]), однако все они ограничиваются нерелятивистским случаем. Один из способов исследования релятивистского случая предложили В. В. Козлов и Е. М. Никишин [6] для пространства Минковского с тремя пространственньми и одной временной координатами, т.е. для $D=3$, где $D$ в дальнейшем - число пространственных координат.

В настоящей статье рассмотрен случай релятивистского двумерного атома водорода (одного электрона в кулоновском поле), т.е. $D=2$.

Основное внимание в работе уделяется радиальным функциям, так как решение для разных размерностей будет отличаться только порядком входящей в него сферической функции. Этим и оправдано исследование двумерного случая, так как уже в нем проявляются все те свойства, которые характерны и всем другим высшим размерностям.

В работеполучено общее решение уравнения и вид волновой функции в координатном пространстве, а также вид волновой функции в импульсном пространстве и значения их энтропий, что является новьми результатами по сравнению с работой [6].

Кроме того, полученные в работе энтропийные соотношения неопределенности сравниваются с принципом Гейзенберга.

2. Частица в центрально-симметричном поле. Рассмотрим релятивистское уравнение Шрёдингера, описьвающее движение одной частищы с массой $m_{0}$ в центрально-симметричном поле:

$$
\left(-E+\frac{\hbar^{2}}{2 m_{0} c^{2}} \square+U(\rho)\right) \Phi=0,
$$

где

$$
\square=\frac{\partial^{2}}{\partial t^{2}}-c^{2}\left(\frac{\partial^{2}}{\partial x^{2}}+\frac{\partial^{2}}{\partial y^{2}}\right)
$$

- оператор Даламбера, т.е. кинетическая энергия частицы, $U(\rho)=U\left(\sqrt{x^{2}+y^{2}-c^{2} t^{2}}\right)$ - потенциальная энергия, $E$ - полная энергия частищы (не включающая в себя внутреннюю энергию), $\hbar$ - постоянная Планка, $c$ - скорость света.

Перейдем к безразмерной системе единищ: $c=1, \hbar=1,2 m_{0}=1$. Тогда рассматриваемое уравнение примет вид

$$
(-E+\square+U(\rho)) \Phi=0 .
$$


Отметим, что потенциальное поле $U(\rho)$ зависит только от координаты $\rho=$ $\sqrt{x^{2}+y^{2}-t^{2}}$, а не от координаты $r=\sqrt{x^{2}+y^{2}}$, как это считалось в других моделях, например, в модели Клейна-Гордона. Это одно из отличий модели Козлова-Никишина от ранее рассматриваемых моделей, которое обеспечивает инвариантность уравнения относительно преобразований Лоренца.

Будем рассматривать решения уравнения в пространственно-подобной области

$$
\mathscr{K}=\left\{\left(x^{2}+y^{2}\right)-t^{2}>0\right\} .
$$

Рассмотрение пространственно-подобных, а не времениподобных, мировых линий другая отличительная особенность модели Козлова-Никишина. Она объясняется тем, что величина $t$ не есть собственное время электрона, это- рассогласование времен электрона и ядра.

Дадим краткую физическую интерпретацию, процитировав некоторые пояснения из комментария Козлова [7] к работе [6].

Попытка релятивистски-инвариантного описания системы взаимодействующих частищ была сделана впервые в квантовой электродинамике, в работах П. А. М. Дирака, В.А. Фока и Б. Подольского 1932 года [8]. Каждой частице приписьвается свое время, отдельное время сопоставляется также электромагнитному полю.

После предложенного в 1946-1948 годах Томонагой и Швингером полевого подхода к квантовой электродинамике многовременной формализм Дирака-Фока-Подольского отошел на второй план. Несмотря на преимущества и успехи полевой теории, в классической релятивистской механике сохранилась проблема непротиворечивого описания динамики взаимодействующих частиц, потому что на этом пути возникают трудности принципиального характера.

В работе [6] предложена релятивистски-инвариантная гамильтонова механика для описания динамики многих частиц. В качестве примера решена задача двух тел с кулоновским потенциалом. Существенное отличие от классической ситуации заключается в том, что теперь точки дискретного спектра становятся бесконечно вырожденными.

Будем искать связанные состояния частищы, т.е. решения уравнения (2), для которых

$$
\int_{\mathscr{K}}|\Phi|^{2} d \mu<\infty,
$$

где $d \mu=d x d y d t$ - элемент объема. Каждой функции $\Phi$ из гильбертова пространства $\mathscr{M}=L^{2}(\mathscr{K})$ можно придать вероятностный смысл. После соответствуюшей нормировки $|\Phi(x, y, t)|^{2}$ означает плотность вероятности обнаружения электрона в точке с относительньми координатами $x, y$ и с собственным временем, отличающимся на $t$ от времени ядра.

Все вычисления волновых функций в координатном представлении будем производить в полной аналогии с вычислениями, вьполненными в работе [6].

В уравнении (2) произведем замену переменных, переходя к сферическо-гиперболическим координатам:

$$
x=\rho \operatorname{ch} \psi \cos \varphi, \quad y=\rho \operatorname{ch} \psi \sin \varphi, \quad t=\rho \operatorname{sh} \psi
$$

где $\rho>0,-\infty<\psi<\infty,-\infty<\varphi<\infty(\bmod 2 \pi)$. 
В новых переменных уравнение принимает вид

$$
\left\{-E-\left[\frac{1}{\rho^{2}} \frac{\partial}{\partial \rho} \rho^{2} \frac{\partial}{\partial \rho}-\frac{1}{\rho^{2} \operatorname{ch} \psi} \frac{\partial}{\partial \psi} \operatorname{ch} \psi \frac{\partial}{\partial \psi}+\frac{1}{\rho^{2} \operatorname{ch}^{2} \psi} \frac{\partial^{2}}{\partial \varphi^{2}}\right]+U(\rho)\right\} \Phi(\rho, \psi, \varphi)=0 .
$$

Мы будем решать это уравнение методом разделения переменных. Для этого положим $\Phi(\rho, \psi, \varphi)=B(\rho, \psi) Y(\varphi)$ и подставим в уравнение. Послепростых преобразований получим

$$
\begin{gathered}
\frac{Y^{\prime \prime}(\varphi)}{Y(\varphi)}=-m^{2} \\
\rho^{2} \operatorname{ch}^{2} \psi\left(U-E-\frac{1}{B(\rho, \psi)}\left(\frac{1}{\rho^{2}} \frac{\partial}{\partial \rho} \rho^{2} \frac{\partial}{\partial \rho}-\frac{1}{\rho^{2} \operatorname{ch} \psi} \frac{\partial}{\partial \psi} \operatorname{ch} \psi \frac{\partial}{\partial \psi}\right) B(\rho, \psi)\right)=-m^{2}
\end{gathered}
$$

где $-m^{2}-$ постоянная разделения.

Тогда

$$
Y(\varphi)=e^{i m \varphi}, \quad m \in \mathbb{Z} .
$$

Продолжим метод разделения переменных и будем искать функцию $B(\rho, \psi)$ в виде

$$
B(\rho, \psi)=\widetilde{T}(\psi) \cdot \widetilde{A}(\rho)
$$

Тогда функции $\widetilde{T}(\psi)$ и $\widetilde{A}(\rho)$ будут удовлетворять соответственно уравнениям

$$
\begin{gathered}
{\left[\frac{1}{\operatorname{ch} \psi} \frac{d}{d \psi} \operatorname{ch} \psi \frac{d}{d \psi}+\frac{m^{2}}{c^{2} \psi}\right] \widetilde{T}=\lambda \widetilde{T}} \\
{\left[\frac{1}{\rho^{2}} \frac{d}{d \rho} \rho^{2} \frac{d}{d \rho}-\left(\frac{\lambda}{\rho^{2}}+U(\rho)-E\right)\right] \widetilde{A}=0,}
\end{gathered}
$$

где $\lambda$ - константа разделения.

Замена $T=\widetilde{T} \sqrt{\operatorname{ch} \psi}$ приводит уравнение (3) к виду

$$
\frac{d^{2}}{d \psi^{2}} T+\left[\frac{(m-1 / 2)(m+1 / 2)}{\operatorname{ch}^{2} \psi}-\left(\lambda+\frac{1}{4}\right)\right] T=0 .
$$

Полученное уравнение на функцию $T$ имеет вид одномерного уравнения Шрёдингера с потенциалом Пошля-Теллера [9]. Спектр уравнения состоит из дискретной части в области $\lambda+1 / 4>0$ и непрерьвной части, заполняющей всю полуось $\lambda+1 / 4 \leqslant 0$. Введем новый спектральный параметр $\nu^{2}=\lambda+1 / 4, \nu>0$. Дискретньй спектр состоит из точек $\nu=1 / 2,3 / 2, \ldots,|m|-1 / 2$. Без ограничения общности можно считать, что $m>0$. Тогда собственные функции имеют вид

$$
T_{\nu, m-1 / 2}(\psi)=\frac{1}{(\operatorname{ch} \psi)^{\nu}} \sum_{k=0}^{m-1 / 2-\nu} \frac{(\nu-m+1 / 2)_{k}(\nu+m+1 / 2)_{k}}{(\nu+1)_{k} k !} \frac{1}{\left(e^{2 \psi}+1\right)^{k}}
$$

где $(p)_{k}=p(p+1) \cdots(p+k-1)$ - символ Похгаммера.

В дальнейшем непрерывньй спектр мы рассматривать не будем и ограничимся рассмотрением только дискретного спектра. Кроме того, необходимо отметить, что при 
$m=0$ дискретньй спектр вообще отсутствует. А в случае $m \neq 0$ спектр состоит из конечного числа точек.

Если ввести обозначение $l=m-1 / 2$, то полученные функции в точности совпадают с собственными функциями для размерности $D=3$. Кроме того, как и в случае $D=3$, где $D$ - число пространственньх координат, замена, приводящая уравнение к необходимому нам виду, одновременно обеспечивает интегрируемость в квадрате полученного решения. Действительно, в сферическо-гиперболических координатах $d \mu=\rho^{2} \operatorname{ch} \psi d \rho d \psi d \varphi$. Таким образом, $\|\Phi\|^{2}=\|Y\|^{2}\|\widetilde{T}\|^{2}\|\widetilde{A}\|^{2}$, где

$$
\begin{gathered}
\|Y\|^{2}=\int_{0}^{2 \pi}|Y|^{2} d \varphi, \quad\|\widetilde{T}\|^{2}=\int_{-\infty}^{\infty}|\widetilde{T}|^{2} \operatorname{ch} \psi d \psi=\int_{-\infty}^{\infty}|T|^{2} d \psi \\
\|\widetilde{A}\|^{2}=\int_{0}^{\infty}|\widetilde{A}|^{2} \rho^{2} d \rho=\int_{0}^{\infty}|A|^{2} d \rho
\end{gathered}
$$

при этом $A(\rho)=\rho \widetilde{A}(\rho)$.

Для функции $A(\rho)$ из (4) получим следующее уравнение:

$$
\frac{d^{2}}{d \rho^{2}} A(\rho)-\left[\frac{(\nu-1 / 2)(\nu+1 / 2)}{\rho^{2}}+U(\rho)-E\right] A(\rho)=0 .
$$

Оно имеет вид одномерного уравнения Шрёдингера с эффективным потенциалом:

$$
U_{\text {эфф }}=U+U_{\text {центр }}
$$

где $U_{\text {центр }}=(\nu-1 / 2)(\nu+1 / 2) / \rho^{2}$ - потенциал центробежных сил.

Таким образом, для любого центрально-симметричного поля волновые функции $Y$ и $T$ не зависят от потенциала, вид которого влияет только на функцию $A$.

В свою очередь полученное уравнение на функцию $A$ полностью повторяет аналогичное уравнение в случае большей размерности $D=3$, что еше раз подтверждает модельность случая $D=2$ и оправдывает его исследования.

3. Решение уравнения в кулоновском поле. Пусть потенциал $U(\rho)$ имеет вид

$$
U(\rho)=\frac{-\varkappa}{\rho}
$$

где $\varkappa$ - положительная постоянная взаимодействия. В размерных величинах $\varkappa=Z e_{0}^{2}$, где $e_{0}-$ заряд электрона, $Z$ - число протонов в ядре водородоподобного атома. В безразмерных величинах считаем $\varkappa=2$. Тогда уравнение на радиальную составляющую имеет вид

$$
\frac{d^{2}}{d \rho^{2}} A(\rho)-\left[\frac{(\nu-1 / 2)(\nu+1 / 2)}{\rho^{2}}+\frac{2}{\rho}-E\right] A(\rho)=0 .
$$

При $E>0$ нет нетривиальных суммируемых с квадратом решений. При $E<0$ введем обозначение $n=1 / \sqrt{-E}$. Тогда уравнение имеет суммируемое с квадратом решение при $n-\nu-1 / 2=k$, где $k=0,1,2, \ldots$ Это решение имеет вид

$$
A_{n, \nu}(\rho)=\rho^{\nu+1 / 2} e^{-\rho / n} L_{k}^{(2 \nu)}\left(\frac{2 \rho}{n}\right)
$$


где $L_{k}^{(2 \nu)}$ - многочлен Лагерра [10].

Таким образом, уровни энергии дискретного спектра вычисляются по формуле Бора

$$
E_{n}=-\frac{1}{n^{2}}, \quad n=1,2,3, \ldots
$$

В соответствии с общепринятой терминологией $n$ - главное квантовое число, $m-$ магнитное квантовое число, $m \in \mathbb{Z} \backslash\{0\}, k$-радиальное квантовое число, $\nu$ квантовое число, не имеюшее аналога в классическом случае и связанное с временной координатой.

Как и в случае большей размерности $D=3$, рассмотренном в работе [6], имеется бесконечное вырождение уровней энергии $E=E_{n}$. Это означает, что одному уровню энергии $E_{n}$ соответствует бесконечное число связанных состояний с определенньми значениями величин $m$ и $\nu$.

Подводя итог, сформулируем следующее

ПРЕДЛОЖЕНИЕ 1. Дискретный спектр уравнения (2) с кулоновским потенииалом $U(\rho)=-2 / \rho$ состоит из әнергетических уровней

$$
E_{n}=-\frac{1}{n^{2}}, \quad n=1,2,3, \ldots,
$$

а соответствующие им собственнье функиии имеют вид

$$
\Phi_{n, \nu, m}(\rho, \psi, \phi)=\frac{C_{A}}{\rho} A_{n, \nu}(\rho) \cdot \frac{C_{T}}{\sqrt{\operatorname{ch} \psi}} T_{\nu, m-1 / 2}(\psi) \cdot C_{Y} Y_{m}(\phi)
$$

$2 \partial e$

$$
\nu=\frac{1}{2}, \frac{3}{2}, \ldots, n-\frac{1}{2}, \quad m= \pm\left(\nu+\frac{1}{2}\right), \pm\left(\nu+\frac{3}{2}\right), \ldots
$$

при этом $Y_{m}(\phi)=e^{i m \phi}$, функиии $T_{\nu, m-1 / 2}(\psi)$ u $A_{n, \nu}(\rho)$ определены формулами $(5)$ и (6) соответственно, а $C_{A}, C_{T}$ и $C_{Y}$ - нормировочные постоянные.

4. Импульсное представление в центрально-симметричном поле. Рассмотрим теперь задачу вычисления преобразования Фурье найденных решений, т.е. найдем выражение волновой функции в импульсном представлении

$$
F(\Phi) \equiv \Phi^{*}\left(p_{x}, p_{y}, p_{t}\right)=\left(\frac{1}{\sqrt{2 \pi}}\right)^{D+1} \int_{\mathscr{K}} \Phi(x, y, t) e^{-i\left(x p_{x}+y p_{y}-t p_{t}\right)} d \mu,
$$

где $p_{x}, p_{y}-$ импульсы, а $p_{t}$ - энергия.

Для вычисления этого интеграла перейдем к сферическо-гиперболическим координатам в сопряженном пространстве:

$$
p_{x}=p \operatorname{ch} \psi^{\prime} \cos \varphi^{\prime}, \quad p_{y}=p \operatorname{ch} \psi^{\prime} \sin \varphi^{\prime}, \quad p_{t}=p \operatorname{sh} \psi^{\prime} .
$$

Тогда

$$
\begin{aligned}
\Phi^{*}\left(p_{x}, p_{y}, p_{t}\right)= & \left(\frac{1}{\sqrt{2 \pi}}\right)^{3} \int_{\mathscr{K}} \widetilde{A}(\rho) \widetilde{T}(\psi) e^{-i p \rho\left(\operatorname{ch}(\psi) \operatorname{ch}\left(\psi^{\prime}\right) \cos \left(\varphi-\varphi^{\prime}\right)-\operatorname{sh}(\psi) \operatorname{sh}\left(\psi^{\prime}\right)\right)} \\
& \times C_{Y} e^{i m \varphi} \rho^{2} \operatorname{ch} \psi d \rho d \psi d \varphi
\end{aligned}
$$


где

$$
\widetilde{A}(\rho)=\frac{C_{A}}{\rho} A_{n, \nu}(\rho), \quad \widetilde{T}(\psi)=\frac{C_{T}}{\sqrt{\operatorname{ch} \psi}} T_{\nu, m-1 / 2}(\psi)
$$

(см. предложение 1).

По формуле Бесселя [11]

$$
\int_{0}^{2 \pi} e^{i m \varphi} e^{-i p \rho \operatorname{ch} \psi \operatorname{ch} \psi^{\prime} \cos \left(\varphi-\varphi^{\prime}\right)} d \varphi=e^{i m \varphi^{\prime}} 2 \pi(-i)^{m} J_{m}\left(p \rho \operatorname{ch} \psi \operatorname{ch} \psi^{\prime}\right) .
$$

В данной работе мы ограничимся случаем $\nu=m-1 / 2$ (невозбужденное состояние); тогда

$$
T_{\nu, \nu}(\psi)=\operatorname{ch}^{-\nu} \psi, \quad \nu \equiv l=m-\frac{1}{2} .
$$

Введем обозначения $a=p \rho \operatorname{ch} \psi^{\prime}, b=p \rho \operatorname{sh} \psi^{\prime}$; тогда получим интеграл

$$
\begin{gathered}
\int_{-\infty}^{\infty} \operatorname{ch}^{-m} \psi J_{m}\left(p \rho \operatorname{ch} \psi^{\prime} \operatorname{ch} \psi\right) \operatorname{ch} \psi e^{i p \rho \operatorname{sh} \psi \operatorname{sh} \psi^{\prime}} d \psi \\
=2 \int_{0}^{\infty} \operatorname{ch}^{-m+1} \psi J_{m}(a \operatorname{ch} \psi) \cos (b \operatorname{sh} \psi) d \psi
\end{gathered}
$$

Сделаем замену $\operatorname{sh} \psi=\zeta$ и воспользуемся следующим равенством:

$$
J_{-1 / 2}(z)=\sqrt{\frac{2}{\pi z}} \cos z
$$

Тогда получим

$$
\sqrt{2 \pi b} \int_{0}^{\infty}\left(\zeta^{2}+1\right)^{-m / 2} J_{m}\left(a \sqrt{\zeta^{2}+1}\right) J_{-1 / 2}(b \zeta) \sqrt{\zeta} d \zeta
$$

- так назьваемьй разрывный интеграл Сонина-Гегенбауэра [11], равньй

$$
\sqrt{2 \pi} \frac{1}{\operatorname{ch}^{m} \psi^{\prime}} \frac{J_{m-1 / 2}(p \rho)}{\sqrt{p \rho}}
$$

Таким образом, мы получили ядро для интегрирования функции $A$, которое не зависит от конкретного вида выбранного потенциала.

А именно, доказано

ПРЕДЛОЖЕНИЕ 2. При $\nu=m-1 / 2$ волновые функиии в импульсном представлении имеют вид

$$
\Phi_{n, \nu, m}^{*}\left(p, \psi^{\prime}, \phi^{\prime}\right)=(-i)^{m} \frac{C_{A}}{p} A_{n, \nu}^{*}(p) \cdot \frac{C_{T}}{\sqrt{\operatorname{ch} \psi^{\prime}}} T_{\nu, \nu}\left(\psi^{\prime}\right) \cdot C_{Y} Y_{m}\left(\phi^{\prime}\right)
$$

где $A_{n, \nu}^{*}(p)$ есть преобразование Фурье-Бесселя

$$
A_{n, \nu}^{*}(p)=\int_{0}^{\infty} A_{n, \nu}(\rho) J_{m-1 / 2}(p \rho) \sqrt{p \rho} d \rho .
$$


Строго говоря, исходя из физических соображений мы должны были бы решать задачу на собственные числа оператора (2) не в гильбертовом пространстве $\mathscr{M}=L^{2}(\mathscr{K})$, а в более узком гильбертовом пространстве $\mathscr{H} \subset \mathscr{M}$, состоящем из функций $f \in \mathscr{M}$, Фурье-преобразование которых $F(f)$ имеет носитель в пространственно-подобной области $\mathscr{K}^{*}=\left\{p_{x}^{2}+p_{y}^{2}-p_{t}^{2}>0\right\}$ пространства импульсов. Тогда преобразование Фурье будет унитарным оператором вида $F: \mathscr{H} \longrightarrow \mathscr{H}^{*}=F \mathscr{H}$. Но определенное подобньп образом пространство $\mathscr{H}$ включает в себя все волновые функции $\Phi_{n, \nu, m}$.

Действительно, формула Сонина-Гегенбауэра гарантирует нам, что преобразование $\Phi$ урье $F(\Phi)$ полученного решения будет равно нулю вне $\mathscr{K}^{*}$, так как выход за пределы этой области в сопряженном пространстве будет означать перестановку параметров $a$ и $b$, но при $a<b$ значение интеграла Сонина-Гегенбауэра будет равно нулю. Поэтому волновые функции $\Phi$ для любого центрально-симметричного поля $U$ принадлежат классу $\mathscr{H}$, что, впрочем, следует также из лоренщ-инвариантной записи преобразования Фурье.

Дальнейшие вычисления зависят от вида решения $A(\rho)$, и в частности, от вида потенциала. Поэтому рассмотрим частный случай - кулоновский потенциал.

5. Преобразование Фурье радиальных волновых функций. Согласно предложениям 1 и 2 нахождение радиальной составляющей преобразования Фурье сводится к вычислению интеграла Фурье-Бесселя:

$$
I(p)=\int_{0}^{\infty} \frac{e^{-\rho / n} \rho^{\nu+1 / 2} L_{k}^{(2 \nu)}(2 \rho / n)}{\rho} \frac{J_{m-1 / 2}(p \rho)}{\sqrt{p \rho}} \rho^{2} d \rho .
$$

Сделаем замену переменных $z=2 \rho / n$ и вспомним, что $\nu=m-1 / 2$. Тогда интеграл преобразуется к виду

$$
I(p)=\left(\frac{n}{2}\right)^{m+2} \int_{0}^{\infty} e^{-z / 2} z^{m+1} L_{k}^{(2 m-1)}(z) \frac{J_{m-1 / 2}(b z)}{\sqrt{b z}} d z
$$

где $k=n-\nu-1 / 2, b=n p / 2$.

Воспользуемся интегральньм представлением функции Бесселя в виде интеграла Пуассона [11]. Тогда

$$
\begin{aligned}
I(p)= & \left(\frac{n}{2}\right)^{m+2} \frac{1}{\Gamma(m)} \frac{1}{\sqrt{\pi}}\left(\frac{b}{2}\right)^{m-1 / 2} \frac{1}{\sqrt{b}} \\
& \times \int_{-1}^{1} d t\left(1-t^{2}\right)^{m-1} \int_{0}^{\infty} e^{-z / 2} z^{2 m} L_{k}^{(2 m-1)}(z) e^{-q z} d z
\end{aligned}
$$

где $q=-i b t$.

Внутренний интеграл можно представить в видепреобразования Лапласа. Мы будем использовать так называемые стандартизованные многочлены Лагерра, которые могут быть определены следующей формулой Родрига:

$$
L_{k}^{(\alpha)}(x) x^{\alpha} e^{-x}=\frac{1}{k !}\left(\frac{d}{d x}\right)^{k}\left\{x^{\alpha+k} e^{-x}\right\}
$$


Тогда

$$
\begin{aligned}
\int_{0}^{\infty} & z^{2 m} L_{k}^{(2 m-1)}(z) e^{-(q+1 / 2) z} d z \\
& =\frac{1}{k !} \frac{\Gamma(2 m+k)}{\Gamma(2 m)} \Gamma(2 m+1) \frac{1}{(q+1 / 2)^{2 m+1}}{ }_{2} F_{1}\left(-k, 2 m+1,2 m, \frac{1}{q+1 / 2}\right) \\
& =\frac{2 m}{k !} \Gamma(2 m+k) \frac{2^{2 m+1}(-1)^{k}}{m} \frac{(1+2 i b t)^{k}}{(1-2 i b t)^{2 m+k}} \frac{k+m+2 i b t m}{1+4 b^{2} t^{2}} .
\end{aligned}
$$

Перепишем $I(p)$ с учетом полученного результата:

$$
I(p)=\frac{2 n^{m+2} b^{m-1}(-1)^{k} \Gamma(2 m+k)}{\sqrt{2 \pi} k ! \Gamma(m)} \cdot \tilde{I}(p),
$$

где

$$
\tilde{I}(p)=\int_{-1}^{1}\left(1-t^{2}\right)^{m-1} \frac{(1+2 i b t)^{k}}{(1-2 i b t)^{2 m+k}} \frac{k+m+2 i b t m}{1+4 b^{2} t^{2}} d t .
$$

Перейдем к новой переменной $\phi$ :

$$
e^{2 i \phi}=\frac{1+2 i b t}{1-2 i b t}, \quad \operatorname{tg} \phi=2 b t
$$

тогда

$\tilde{I}(p)=\frac{b}{\left(4 b^{2}\right)^{m}} \int_{-\phi_{0}}^{\phi_{0}}\left(\operatorname{tg}^{2} \phi_{0}-\operatorname{tg}^{2} \phi\right)^{m-1} e^{2 i n \phi} \cos ^{2 m-1} \phi\left((n+m) e^{i \phi}+(n-m) e^{-i \phi}\right) d \phi$,

где $n=k+m, \operatorname{tg} \phi_{0}=2 b$.

Дальнейшее решение задачи может проходить по двум направлениям. Во-первых, можно изучать асимптотическое поведение решений при $n \rightarrow \infty$. Такое асимптотическое представление волновой функции необходимо для изучения системы в квазиклассическом приближении. Во-вторых, можно поставить вопрос о точном вычислении интеграла. Такие результаты необходимы для изучения основного невозбужденного состояния атома (и, возможно, нескольких первых возбужденных состояний).

В рамках данной работы нас прежде всего будет интересовать поведение системы в квазиклассическом приближении. Поэтому вначале мы остановимся на первом направлении. В конце раздела мы приведем некоторые результаты и по второму направлению исследований.

Проинтегрируем полученньй интеграл $m$ раз по частям. Легко видеть, что за счет первого множителя подьнтегральной функции подстановка в концах отрезка интегрирования будет давать 0. А значит, первьй ненулевой член и даст нам искомую асимптотику.

Запишем

$$
\tilde{I}(p)=\frac{b \cos ^{2} \phi_{0}}{\sin ^{2 m} \phi_{0}} \int_{-\phi_{0}}^{\phi_{0}}\left(\sin \left(\phi_{0}-\phi\right) \sin \left(\phi_{0}+\phi\right)\right)^{m-1} e^{2 i n \phi} \cos \phi\left((n+m) e^{i \phi}+(n-m) e^{-i \phi}\right) d \phi .
$$


Введем обозначения

$$
P=\sin \left(\phi_{0}-\phi\right) \sin \left(\phi_{0}+\phi\right), \quad Q=\cos \phi\left((n+m) e^{i \phi}+(n-m) e^{-i \phi}\right) .
$$

Тогда, учитьвая предыдущее замечание об интегрировании по частям, имеем

$$
\tilde{I}(p)=\frac{b \cos ^{2} \phi_{0}}{\sin ^{2 m} \phi_{0}}\left(\frac{-1}{2 i n}\right)^{m-1} \int_{-\phi_{0}}^{\phi_{0}} e^{2 i n \phi} \frac{d^{m-1}}{d \phi^{m-1}}\left(P^{m-1} Q\right) d \phi .
$$

Проинтегрируем полученный интеграл по частям еще раз:

$$
\begin{aligned}
\hat{I}(p) & =\int_{-\phi_{0}}^{\phi_{0}} e^{2 i n \phi} \frac{d^{m-1}}{d \phi^{m-1}}\left(P^{m-1} Q\right) d \phi \\
& =\left.\frac{1}{2 i n} e^{2 i n \phi} \frac{d^{m-1}}{d \phi^{m-1}}\left(P^{m-1} Q\right)\right|_{-\phi_{0}} ^{\phi_{0}}-\frac{1}{2 i n} \int_{-\phi_{0}}^{\phi_{0}} e^{2 i n \phi} \frac{d^{m}}{d \phi^{m}}\left(P^{m-1} Q\right) d \phi .
\end{aligned}
$$

Нас интересует первый член полученной разности, так как он определяет асимптотику всего интеграла.

Легко видеть, что единственным ненулевым слагаемьм полученной производной после подстановки в концах интегрирования будет

$$
\frac{d^{m-1}}{d \phi^{m-1}}\left(P^{m-1}\right) Q=(m-1) !\left(P^{\prime}\right)^{m-1} Q .
$$

После несложных вычислений в случае четного $m$ окончательно получаем

$$
I_{\text {четн. }}(p) \approx \frac{2 n^{m+2}(-1)^{k} \Gamma(2 m+k)}{\sqrt{2 \pi} k !} \frac{\cos ^{2} \phi_{0}}{\sin 2 \phi_{0}}\left(\frac{-1}{2 i n}\right)^{m-1} \frac{-4 n \cos ^{2} \phi_{0}}{2 i n} \cos 2 n \phi_{0} .
$$

Аналогичная формула имеет место и для нечетных $m$ :

$$
I_{\text {нечетн. }}(p) \approx \frac{2 n^{m+2}(-1)^{k} \Gamma(2 m+k)}{\sqrt{2 \pi} k !} \frac{\cos ^{2} \phi_{0}}{\sin 2 \phi_{0}}\left(\frac{-1}{2 i n}\right)^{m-1} \frac{4 i n \cos ^{2} \phi_{0}}{2 i n} \sin 2 n \phi_{0} .
$$

Осталось умножить полученные выражения на нормировочную константу $C_{A}$, чтобы получить окончательный ответ.

Константа $C_{A}$ находится из условия нормировки

где

$$
C_{A}^{2} \int_{0}^{\infty}|\widetilde{A}|^{2} \rho^{2} d \rho=1
$$

$$
\widetilde{A}(\rho)=\frac{C_{A}}{\rho} A_{n, \nu}(\rho)
$$

и $A_{n, \nu}(\rho)$ определена формулой (6). Воспользовавшись формулой Родрига (8), используя соотношения ортогональности для многочленов Лагерра и формулу интегрирования по частям, после несложных вычислений получаем

$$
C_{A}^{2}=\frac{2^{2 m}}{n^{2 m+2}} \frac{(n-m) !}{(n+m-1) !} .
$$

Тогда

$$
\widetilde{A}_{n, \nu}^{*}(p) \approx i^{m}(-1)^{k} \frac{4}{\sqrt{2 \pi}} \frac{1}{n^{m-2}} \sqrt{\frac{(n+m-1) !}{(n-m) !}} \frac{\cos ^{4} \phi_{0}}{\sin 2 \phi_{0}} j_{n, m}\left(\phi_{0}\right)
$$

где

$$
j_{n, m}\left(\phi_{0}\right)= \begin{cases}2 \cos \left(2 n \phi_{0}\right), & m \text { четное } \\ -2 i \sin \left(2 n \phi_{0}\right), & m \text { нечетное. }\end{cases}
$$

Осталось воспользоваться формулой Стирлинга. Тогда получим 
ПРЕДЛОЖЕНИЕ 3. Для радиальной волновой функиии в импульсном представлении равномерно на всем промежутке $0<p<+\infty$ при $n \rightarrow \infty$ справедлива асимптотическая формула

$$
\widetilde{A}_{n, \nu}^{*}(p)=(-i)^{m}(-1)^{n} \frac{4}{\sqrt{2 \pi}} n^{3 / 2} \frac{\cos ^{4} \phi_{0}}{\sin 2 \phi_{0}} j_{n, m}\left(\phi_{0}\right)\left[1+O\left(\frac{1}{n}\right)\right],
$$

где $j_{n, m}\left(\phi_{0}\right)$ определена формулой $(10) u \phi_{0}=\operatorname{arctg}(n p)$.

Если же стоит задача точного вычисления интеграла (7), мы можем в формуле (9) разложить $\left(\operatorname{tg}^{2} \phi_{0}-\operatorname{tg}^{2} \phi\right)^{m-1}$ по степеням $\operatorname{tg}^{2} \phi$ и воспользоваться следующим результатом:

$$
\begin{aligned}
\int \operatorname{tg}^{p} \phi \cos ^{q} \phi e^{i r \phi} d \phi= & \frac{i}{q-r} e^{i r \phi}\left(1-e^{2 i \phi}\right)^{-p}\left(1+e^{2 i \phi}\right)^{p-q} \\
& \times F_{1}\left(\frac{r-q}{2}, p-q,-p, \frac{r-q}{2}+1,-e^{2 i \phi}, e^{2 i \phi}\right) \cos ^{q} \phi \operatorname{tg}^{p} \phi,
\end{aligned}
$$

где $F_{1}$ - гипергеометрическая функция Аппеля.

Таким образом, полученный интеграл полностью вычисляется.

Рассмотрим простейший частньй случай.

Положим $m=1$. Тогда интеграл примет вид

$$
I(p)=\left(\frac{n}{2}\right)^{3} \int_{0}^{\infty} e^{-z / 2} z^{2} L_{k}^{(1)}(z) \frac{J_{1 / 2}(b z)}{\sqrt{b z}} d z .
$$

Записьвая $J_{1 / 2}(z)=\sqrt{2 /(\pi z)} \sin z$ и используя формулу для преобразования Лапласа, получаем

$$
\widetilde{A}_{n, \nu}^{*}(p)=(-1)^{n-1} \frac{8}{\sqrt{2 \pi}} n^{3 / 2} \frac{\cos ^{4} \phi_{0} \sin 2 n \phi_{0}}{\sin 2 \phi_{0}},
$$

что совпадает с главным членом асимптотики.

Теперь мы можем зафиксировать вакуумные волновые функции $\Phi_{V}$ и $\Phi_{V}^{*}$ для основного состояния, т.е. при $n=1, \nu=1 / 2, m= \pm 1$ в координатном и импульсном представлениях соответственно.

ПРЕДЛОЖЕНИЕ 4. В основном состоянии

$$
\Phi_{V}=2 e^{-\rho} \cdot \frac{1}{\sqrt{\pi}} \frac{1}{\operatorname{ch} \psi} \frac{e^{ \pm i \varphi}}{\sqrt{2 \pi}}, \quad \Phi_{V}^{*}=\frac{4 \sqrt{2}}{\sqrt{\pi}} \frac{1}{\left(1+p^{2}\right)^{2}} \cdot \frac{1}{\sqrt{\pi}} \frac{1}{\operatorname{ch} \psi^{\prime}} \frac{e^{ \pm i \varphi^{\prime}}}{\sqrt{2 \pi}} .
$$

6. Вычисление энтропии в координатном представлении. Если волновая функция $\Phi$ нормирована: $\|\Phi\|^{2}=1$, то информационная энтропия Больцмана-Шеннона определяется формулой

$$
S_{\Phi}=-\int_{\mathscr{K}}|\Phi|^{2} \ln |\Phi|^{2} d \mu .
$$

Представим волновую функцию в виде $\Phi=\widetilde{A}(\rho) \cdot \widetilde{T}(\psi) \cdot \widetilde{Y}(\varphi)$ (см. предложение 1$)$. 
Тогда $S_{\Phi}=S_{Y}+S_{T}+S_{A}$, где

$$
\begin{gathered}
S_{Y}=-\int_{0}^{2 \pi}|\widetilde{Y}|^{2} \ln |\widetilde{Y}|^{2} d \varphi, \quad S_{T}=-\int_{-\infty}^{\infty}|\widetilde{T}|^{2} \ln |\widetilde{T}|^{2} \operatorname{ch} \psi d \psi, \\
S_{A}=-\int_{0}^{\infty}|\widetilde{A}|^{2} \ln |\widetilde{A}|^{2} \rho^{2} d \rho .
\end{gathered}
$$

Легко видеть, что

$$
S_{Y}=-\int_{0}^{2 \pi} C_{Y}^{2} \ln C_{Y}^{2} d \varphi=\ln 2 \pi .
$$

Для дальнейших вычислений введем обозначение

$$
N(\tau)=\int_{-\infty}^{\infty}|\widetilde{T}|^{\tau} \operatorname{ch} \psi d \psi
$$

тогда

$$
S_{T}=-\left.2 \frac{\partial}{\partial \tau} \ln N(\tau)\right|_{\tau=2} .
$$

Этот метод вычисления энтропии детально обоснован в работах [2], [12].

Вычислим $N(\tau)$ :

$$
N(\tau)=2 C_{T}^{\tau} \int_{0}^{\infty} \operatorname{ch}^{1-m \tau} \psi d \psi .
$$

Для нахождения интеграла сделаем замену переменных $z=1 / \operatorname{ch}^{2} \psi$; тогда интеграл преобразуется к виду

$$
\begin{aligned}
N(\tau) & =2 C_{T}^{\tau} \int_{0}^{1} z^{(m \tau-1) / 2} \frac{d z}{2 z \sqrt{1-z}}=C_{T}^{\tau} \int_{0}^{1} z^{(m \tau-3) / 2}(1-z)^{-1 / 2} d z \\
& =C_{T}^{\tau} B\left(\frac{m \tau-1}{2}, \frac{1}{2}\right) .
\end{aligned}
$$

Следовательно,

$$
\begin{aligned}
S_{T} & =\ln \frac{1}{C_{T}^{2}}+m\left(\psi(m)-\psi\left(m-\frac{1}{2}\right)\right) \\
& =\ln B\left(m-\frac{1}{2}, \frac{1}{2}\right)+m\left(\psi(m)-\psi\left(m-\frac{1}{2}\right)\right) .
\end{aligned}
$$

Здесь $B$ и $\psi$ - бета-функция и логариффмическая производная гамма-функции соответственно.

Аналогично, если

$$
N(\tau)=\int_{0}^{\infty}|\widetilde{A}|^{\tau} \rho^{2} d \rho
$$

то

$$
S_{A}=-\left.2 \frac{\partial}{\partial \tau} \ln N(\tau)\right|_{\tau=2} .
$$


Для вычисления интеграла воспользуемся асимптотическим выражением многочленов Лагерра. Нам понадобится равномерная асимптотика типа Планшереля-Ротаха на интервале осцилляпий. Вкратце напомним вывод этой формулы с целью получить асимптотику в удобном для нас виде. Для этого представим многочлен Лагерра

$$
L_{k}^{(2 m-1)}(x)=x^{-(2 m-1)} e^{x} \frac{1}{k !}\left(\frac{d}{d x}\right)^{k}\left\{x^{k+2 m-1} e^{-x}\right\}
$$

с помошью формулы Коши в виде

$$
L_{k}^{(2 m-1)}(x)=\frac{e^{x}}{x^{2 m-1}} \cdot \frac{1}{2 \pi i} \oint_{(x)} \frac{\xi^{k+2 m-1} e^{-\xi} d \xi}{(\xi-x)^{k+1}} .
$$

Положим $\Lambda_{n}(x)=L_{k}^{(2 m-1)}(4 n x)$. Тогда

$$
\Lambda_{n}(x)=\frac{e^{4 n x}}{x^{2 m-1}} \cdot \frac{1}{2 \pi i} \oint_{(x)} \frac{\xi^{k+2 m-1} e^{-4 n \xi} d \xi}{(\xi-x)^{k+1}},
$$

где $n=k+m$.

Воспользуемся методом перевала. Введем обозначения

$$
F=\frac{\xi e^{-4 \xi}}{\xi-x}, \quad G=\ln F, \quad f=\frac{\xi^{2 m-1} e^{-4 m \xi}}{\xi-x}
$$

и вычислим критические точки, в которых

$$
\frac{\partial}{\partial \xi} G=\frac{1}{\xi}-4-\frac{1}{\xi-x}=0 .
$$

Отсюда находим

$$
\xi_{*}=\frac{x+\sqrt{x(x-1)}}{2}, \sqrt{x(x-1)} \sim x \quad \text { при } \quad x \rightarrow \infty .
$$

Тогда

$$
\begin{gathered}
\Lambda_{n}(x)=\frac{e^{4 n x}}{x^{2 m-1}} \cdot \frac{1}{2 \pi i} \cdot F_{*}^{n-m} \cdot \sqrt{-\frac{2 \pi}{n G_{*}^{\prime \prime}}}\left(f_{*}+O\left(\frac{1}{n}\right)\right) \quad \text { при } n \rightarrow \infty, \\
F_{*}=\left.F\right|_{\xi=\xi_{*}}=-\frac{(x+\sqrt{x(x-1)})^{2}}{x} e^{-2(x+\sqrt{x(x-1)})} \\
G_{*}^{\prime \prime}=\left.G_{*}^{\prime \prime}\right|_{\xi=\xi_{*}}=\frac{16}{x} \sqrt{x(x-1)}, \quad f_{*}=-\frac{x^{m-1}(\sqrt{x}+\sqrt{(x-1)})^{2 m} e^{-2 m(x+\sqrt{x(x-1)})}}{2^{2 m-2}},
\end{gathered}
$$

откуда окончательно и получаем асимптотическое выражение для $\Lambda_{n}$ :

$$
\begin{gathered}
\Lambda_{n}(x) \sim(-1)^{n-m} \frac{1}{4 \sqrt{2 \pi n}} \frac{\sqrt{x}(\sqrt{x}+\sqrt{x-1})^{2 n}}{\sqrt{\sqrt{x(x-1)}}} e^{2 n(x-\sqrt{x(x-1)}} \frac{1}{x^{m} 2^{2 m-2}}, \\
n \rightarrow \infty, \quad x \in \mathbb{C} \backslash[0,1] .
\end{gathered}
$$


Пусть $x \in(0,1)$; тогда если $x=x+i \cdot 0$, то $\sqrt{x-1}=i \sqrt{1-x}$. Положим $x=\cos ^{2} \frac{\phi}{2}$, $0<\phi<\pi$; тогда $\sqrt{1-x}=\sin (\phi / 2)$. Окончательно имеем

$$
\begin{aligned}
\Lambda_{n}(x) \sim & (-1)^{n-m} \frac{1}{4 \sqrt{2 \pi}} \frac{1}{\sqrt{n}}\left(\cos \frac{\phi}{2}\right)^{1 / 2-2 m}\left(\sin \frac{\phi}{2}\right)^{-1 / 2} e^{2 n \cos ^{2}(\phi / 2)} \\
& \times \frac{1}{2^{2 m-2}} 2 \cos \left\{n(\phi-\sin \phi)-\frac{\pi}{4}\right\}
\end{aligned}
$$

Это формула типа Планшереля-Ротаха.

Для волновой функции $\widetilde{A}(\rho)$ имеем

$$
\widetilde{A}(\rho)=C_{A} e^{-\rho / n} \rho^{m-1} L_{k}^{(2 m-1)}\left(\frac{2 \rho}{n}\right) .
$$

В интеграле (11) сделаем замену переменных $x=2 \rho / n$. Тогда

$$
N(\tau)=C_{A}^{\tau}\left(\frac{n}{2}\right)^{(m-1) \tau+3} \cdot \tilde{N}(\tau) d x
$$

где

$$
\tilde{N}(\tau)=\int_{0}^{\infty}\left|e^{-x / 2} x^{m-1} L_{k}^{(2 m-1)}(x)\right|^{\tau} x^{2} d x
$$

Далее, $\tilde{N}(\tau)=(4 n)^{3+\tau(m-1)} \cdot \bar{N}(\tau) d x$, где

$$
\bar{N}(\tau)=\int_{0}^{\infty}\left|e^{-2 n x} x^{m-1} \Lambda_{n}(x)\right|^{\tau} x^{2} d x
$$

Воспользуемся полученньм ранее асимптотическим выражением для многочлена Лагерра:

$$
\bar{N}(\tau) \approx\left\{\frac{1}{2^{2 m-1} \sqrt{2 \pi n}}\right\}^{\tau} \int_{0}^{\pi}\left(\cos \frac{\phi}{2}\right)^{5-3 \tau / 2}\left(\sin \frac{\phi}{2}\right)^{1-\tau / 2}\left|\cos \left(n \gamma-\frac{\pi}{4}\right)\right|^{\tau} d \phi
$$

где $\gamma=\gamma(\phi)=\phi-\sin \phi$.

Пусть

$$
\begin{gathered}
N^{*}(\tau)=\int_{0}^{\pi}\left(\cos \frac{\phi}{2}\right)^{5-3 \tau / 2}\left(\sin \frac{\phi}{2}\right)^{1-\tau / 2}\left|\cos \left(n \gamma-\frac{\pi}{4}\right)\right|^{\tau} d \phi \\
g_{\tau}(\phi)=\left(\cos \frac{\phi}{2}\right)^{5-3 \tau / 2}\left(\sin \frac{\phi}{2}\right)^{1-\tau / 2}, \\
G(\tau)=\int_{0}^{\pi} g_{\tau}(\phi) \frac{d \phi}{\pi}, \quad C(\tau)=\int_{0}^{\pi}|\cos \phi|^{\tau} \frac{d \phi}{\pi}
\end{gathered}
$$

Тогда по лемме из работы [12] $N^{*}(\tau) \rightarrow \pi \cdot G(\tau) \cdot C(\tau)$ при $n \rightarrow \infty$. По формуле Стирлинга

$$
C_{A} \sim \frac{2^{m}}{n^{2 m} \sqrt{n}} \quad \text { при } n \rightarrow \infty \text {. }
$$


Окончательно,

$$
N(\tau) \rightarrow\left(\frac{1}{\sqrt{2 \pi}} \frac{1}{n^{3}}\right)^{\tau} 8 n^{6} \pi G(\tau) C(\tau) \quad \text { при } n \rightarrow \infty
$$

Вычислим значения $G(\tau)$ и $C(\tau)$, входящие в формулу:

$$
G(\tau)=\frac{1}{\pi} B\left(3-\frac{3 \tau}{4}, 1-\frac{\tau}{4}\right), \quad C(\tau)=\frac{1}{\pi} B\left(\frac{\tau+1}{2}, \frac{1}{2}\right) .
$$

Тогда

$$
S_{A}=-\left.2 \frac{\partial}{\partial \tau} \ln N(\tau)\right|_{\tau=2}=6 \ln n-\ln 2+\ln \pi+o(1) .
$$

Таким образом, нами доказано

ПРЕДЛОЖЕНИЕ 5. 1) При $\nu=m-1 / 2$ волновая функиия в координатном представлении $\Phi_{n, \nu, m}$ имеет следующую информационную әнтропию:

$$
S\left(\Phi_{n, \nu, m}\right)=S_{Y}+S_{T}+S_{A}
$$

əวe

$$
S_{Y}=\ln 2 \pi, \quad S_{T}=\ln B\left(m-\frac{1}{2}, \frac{1}{2}\right)+m\left(\psi(m)-\psi\left(m-\frac{1}{2}\right)\right) .
$$

2) Информационная әнтропия радиальной волновой функции имеет следующую коротковолновую асимптотику:

$$
S_{A}=6 \ln n-\ln 2+\ln \pi+o(1) \quad n p u \quad n \rightarrow \infty .
$$

3) Информачионная энтропия вакуума равна

$$
S\left(\Phi_{V}\right)=\ln 2+2 \ln \pi+3 .
$$

Утверждение 3) является прямым следствием предложения 4.

Ниже мы вычислим энтропию в импульсном представлении и проинтерпретируем полученные результаты в связи с принципом неопределенности.

7. Вычисление энтропии в импульсном представлении. Вычислим энтропию

$$
S_{\Phi^{*}}=-\int_{\mathscr{K}^{*}}\left|\Phi^{*}\right|^{2} \ln \left|\Phi^{*}\right|^{2} d \mu^{*}
$$

Волновая функция имеет вид $\Phi^{*}=\widetilde{A}^{*}(p) \cdot \widetilde{T}^{*}\left(\psi^{\prime}\right) \cdot \widetilde{Y}^{*}\left(\varphi^{\prime}\right)$ (см. предложение 2$)$.

Тогда $S_{\Phi^{*}}=S_{Y^{*}}+S_{T^{*}}+S_{A^{*}, \text { где }}$

$$
S_{Y^{*}}=S_{Y}, \quad S_{T^{*}}=T_{Y}, \quad S_{A^{*}}=-\int_{0}^{\infty}\left|\widetilde{A}^{*}\right|^{2} \ln \left|\widetilde{A}^{*}\right|^{2} p^{2} d p .
$$

Пусть

$$
N(\tau)=\int_{0}^{\infty}\left|\widetilde{A}^{*}\right|^{\tau} p^{2} d p
$$


тогда

$$
S_{A^{*}}=-\left.2 \frac{\partial}{\partial \tau} \ln N(\tau)\right|_{\tau=2} .
$$

Воспользуемся предложением 3. Пусть сначала $m$ нечетное. Тогда

$$
N(\tau) \approx\left(\frac{4}{\sqrt{2 \pi}} n^{3 / 2}\right)^{\tau} \int_{0}^{\infty}\left|\frac{\cos ^{4} \phi}{\sin 2 \phi} 2 \sin 2 n \phi\right|^{\tau} p^{2} d p, \quad \phi=\operatorname{arctg} n p .
$$

Следовательно,

$$
N(\tau) \approx\left(\frac{4}{\sqrt{2 \pi}} n^{3 / 2}\right)^{\tau} n^{-3} \int_{0}^{\pi / 2}(\sin \phi)^{2-\tau}|\sin 2 n \phi|^{\tau}(\cos \phi)^{3 \tau-4} d \phi .
$$

Введем обозначение

$$
N^{*}(\tau)=\int_{0}^{\pi / 2}(\sin \phi)^{2-\tau}|\sin 2 n \phi|^{\tau}(\cos \phi)^{3 \tau-4} d \phi .
$$

Последний интеграл при $n \rightarrow \infty$ можно представить в виде

$$
N^{*}(\tau) \rightarrow \frac{\pi}{2} \int_{0}^{\pi}(\sin \phi)^{\tau} \frac{d \phi}{\pi} \cdot \int_{0}^{\pi}(\sin \phi)^{2-\tau}|\cos \phi|^{3 \tau-4} \frac{d \phi}{\pi}
$$

Тогда

$$
N^{*}(\tau) \sim \frac{1}{2 \pi} B\left(\frac{\tau+1}{2}, \frac{1}{2}\right) B\left(\frac{3-\tau}{2}, \frac{3 \tau-3}{2}\right)
$$

а значит,

$$
S_{A^{*}}=-3 \ln n+3 \ln 2+\ln \pi-5 .
$$

Очевидно, что для четных $m$ результат будет тем же.

Тем самым, справедливо

ПРЕДЛОЖЕНИЕ 6. 1) При $\nu=m-1 / 2$ волновая функиия в импульсном представлении $\Phi_{n, \nu, m}^{*}$ имеет следующую информационную әнтропию:

$$
S\left(\Phi_{n, \nu, m}^{*}\right)=S_{Y^{*}}+S_{T^{*}}+S_{A^{*}}
$$

əде $S_{Y^{*}}=S_{Y}, S_{T^{*}}=S_{T}$.

2) Информаиионная әнтропия радиальной волновой функиии имеет следующую коротковолновую асимптотику:

$$
S_{A^{*}}=-3 \ln n+7 \ln 2+\ln \pi-5+o(1) \quad n p u \quad n \rightarrow \infty .
$$

3) Информачионная энтропия вакуума равна

$$
S\left(\Phi_{V}^{*}\right)=6 \ln 2+3 \ln \pi-\frac{10}{3} .
$$

В заключение обсудим энтропийные соотношения неопределенности [1]

$$
S_{\Phi}+S_{\Phi^{*}} \geqslant(D+1)(1+\ln \pi),
$$


где $D+1$ - полная размерность всего пространства, а $D$ - число пространственных координат.

Рассмотрим это условие в случае $n=1$. Для этого воспользуемся точными значениями энтропий из предложений 5 и 6 :

$$
S_{\Phi_{V}}=3+\ln 2+2 \ln \pi, \quad S_{\Phi_{V}^{*}}=3 \ln \pi+6 \ln 2-\frac{10}{3} .
$$

Таким образом,

$$
S_{\Phi_{V}}+S_{\Phi_{V}^{*}}=5 \ln \pi+7 \ln 2-\frac{1}{3} \approx 10.2423
$$

Очевидно, что

$$
S_{\Phi_{V}}+S_{\Phi_{V}^{*}} \geqslant 3(1+\ln \pi) \approx 6.4342
$$

Сравним полученные результаты с принципом неопределенности Гейзенберга, которьй в данном случае утверждает, что

$$
\Delta \rho \cdot \Delta p \geqslant \frac{3}{2}
$$

где

$$
(\Delta \rho)^{2}=\left\langle\rho^{2}\right\rangle=\int_{\mathscr{K}} \rho^{2}\left|\Phi_{V}\right|^{2} d \mu, \quad(\Delta p)^{2}=\left\langle p^{2}\right\rangle=\int_{\mathscr{K}^{*}} p^{2}\left|\Phi_{V}^{*}\right|^{2} d \mu^{*} .
$$

Простые вычисления показьвают, что

$$
\Delta \rho=\sqrt{3}, \quad \Delta p=1
$$

Таким образом, неравенство Гейзенберга (12) безусловно вьполнено.

Сравним, насколько велико отклонение величин $\Delta \rho \cdot \Delta p$ с одной стороны, и $S_{\Phi}+S_{\Phi *}$ с другой от своих минимальных значений. Мы видим, что

$$
\frac{\Delta \rho \cdot \Delta p}{3 / 2} \approx 1.1547, \quad \frac{S_{\Phi}+S_{\Phi^{*}}}{(D+1)(1+\ln \pi)} \approx 1.5919
$$

Итак, второе отношение больше, а это означает, что энтропия сильнее реагирует на неопределенность квантово-механической системы, чем принцип неопределенности Гейзенберга.

Если же $n \rightarrow \infty$, то

$$
S_{\Phi}+S_{\Phi^{*}} \sim 3 \ln n \rightarrow \infty,
$$

а значит, очевидньм образом

$$
S_{\Phi}+S_{\Phi^{*}} \geqslant 3(1+\ln \pi)
$$

при больших $n$. 


\section{СПИСОК ЦИТИРОВАННОЙ ЛИТЕРАТУРЫ}

[1] Bialynicki-Birula I., Mycielski J. Uncertainty relations for information entropy in wave mechanics // Cumm. Math. Phys. 1975. V. 44. P. 129-132.

[2] Dehesa J.S., Yáñez R. J., Aptekarev A.I., Buyarov V. Strong asymptotics of Laguerre polynomials and information entropies of two-dimensional harmonic oscillator and one-dimensional Coulomb potentials // J. Math. Phys. 1998. V. 39. №6. P. 3050-3060.

[3] Yáñez R.J., Van Assche W., Dehesa J.S. Position and momentum information entropies of the $D$-dimensional harmonic oscillator and hydrogen atom // Phys. Rev. A. 1994. V. 50. P. 3065-3079.

[4] Dehesa J.S., Martinez-Finkelshtein A., Sorokin V. N. Quantum-information entropies for highly excited states of single-particle systems with power-type potentials // Phys. Rev. A. 2002. V. 66. P. 2109.

[5] Dehesa J.S., Martinez-Finkelshtein A., Sorokin V. N. Asymptotics of information entropies of some Toda-like potentials // J. Math. Phys. 2003. V. 44. № 1. P. 36-47.

[6] Козлов В.В., Никишин Е.М. Релятивистский вариант гамильонова формализма и волновые функции водородоподобного атома // Вестн. МГУ. Сер. 1. Матем., мех. 1986. № 5 . C. $11-20$.

[7] Козлов В.В. Релятивистская задача многих тел и ее квантование // Избранные вопросы математического анализа / ред. Е. М. Никишин. Доклады по математике и ее приложениям. Т. 3. Вьп. 1. М.-Тула, 1990. С. 430-431.

[8] Фок В. А. Работы по квантовой теории поля. Л.: Изд-во Ленингр. ун-та, 1957.

[9] Флюгге З. Задачи по квантовой механике. М.: Мир, 1974.

[10] Никифоров А.Ф., Уваров В. Б. Специальные функции математической физики (2-е изд.). М.: Наука, 1984.

[11] Бейтмен Г., Эрдейи А. Высшие трансцендентные функции. М.: Наука, 1965.

[12] Аптекарев А.И., Буяров В. С., Дегеза Х. С. Асимптотическое поведение $L^{p}$-норм и энтропии для общих ортогональных многочленов // Матем. сб. 1995. Т. 82. С. 373-395.

Московский государственный университет им. М.В. Ломоносова

E-mail: spex19@mail.ru

Поступило

07.02 .2005

Исправленный вариант

30.03.2005 